\title{
Supervisión en la determinación del riesgo bancario $^{1}$
}

\author{
Mujica F., Aníbal J. " \\ Jiménez Medina, Edinson

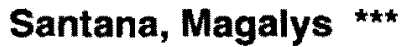 \\ Portillo, Ninoska
}

\section{Resumen}

El presente trabajo tiene como finalidad describir y aplicar el modelo de supervisión utilizado por la Superintendencia de Bancos en Venezuela para monitorear la gestión de las actividades de la banca, el estudio en cuestión se concretará en abordar la metodología de supervisión extra situ aplicada para evaluar el riesgo bancario. Para el análisis en referencia, se utilizó como población la totalidad de la banca comercial y universal vigente para el periodo comprendido desde el primer semestre de 1996 hasta el primer semestre de 1998, extrayéndose una muestra de aquellas instituciones con mayor volumen de captación de ahorros. El tipo de estudio es de carácter descriptivo. Como resultado principal del estudio se puede afirmar que el $57 \%$ de las instituciones bancarias tuvieron un desempeño financiero insatisfactorio, lo cual permite concluir la existencia de una banca con una

\section{Recibido: 99-09-28. Aceptado: 00-05-23}

* Licenciado en Contaduría Pública. Maestría en Finanzas. Investigador del Centro de Estudios de la Empresa de la Facultad de Ciencias Económicas y Sociales de La Universidad del Zulia. Maracaibo. Venezuela. Apartado Postal 526. Telf. 5861-596557. Fax: 5861-416025.

E-mail:anibalmujica@ hotmail.com

** Economista. Maestría en Finanzas. Investigador del Centro de Estudios de la Empresa de la Facultad de Ciencias Económicas y Sociales de La universidad del Zulia.

E-mail: ejimenez72 hotmail.com.

*** Socióloga.

**** Economista.

1 Resultados de Proyecto de Investigación financiado por el Consejo de Desarroilo Clentífico y Humanístico de LUZ. 
valoración de riesgo que asume un comportamiento de moderado a medianamente alto, lo que evidencia que la crisis reciente aún no ha sido superada por el sector bancario.

Palabras clave: Supervisión de Riesgo, Valoración del Riesgo, Banca Comercial, Banca Universal.

\section{A Model of Supervision in the Determination of Bank Risk}

\section{Abstract}

The objective of this paper is to describe and apply the model of supervision used by the Superintendent of Banking in order to monitor the management of banking activities. The study concentrates on the methodology of supervision extra situ applied in the evaluation of banking risk. For this analysis, the population used was the entire universe of commercial and universal banks that were functioning from the first period of 1996 to the first period of 1998. A sample was extracted of those banks with greater volumes of savings accounts. The study was descriptive in nature. The principal result of the study was the affirmation that $57 \%$ of the banking institutions had unsatisfactory financial management practices, which leads to the conclusion that the banking sector presents moderate to slightly high risk behavior. This also indicates that the recent banking crisis has not yet been overcome by the banking sector.

Key words: Risk supervision, risk evaluation, commercial banking, the universal bank.

\section{Introducción}

El sistema financiero venezolano confrontó a principio de 1994 una profunda crisis, que afectó no sólo a las entidades bancarias directamente involucradas, sino que ha tenido consecuencias sobre diversos grupos financieros. A partir de entonces, las autoridades han nacionalizado, intervenido y/o cerrado una cantidad considerable del conjunto de intermediarios financieros.

La crisis ha demostrado que el sistema financiero venezolano presenta deficiencias coyunturales y estructurales, que han sido originadas por diversos factores. Faraco lo expresa cuando señala que:

"... la génesis de las crisis bancarias se suele atribuir a la confluencia de diversos factores endógenos o microeconómicos y exógenos o macroeconómicos. Los primeros comprenden la gestión deficiente y el fraude y autopréstamo, aspectos sobre los cuales la institución puede y debe ejercer un grado significativo de control. Los segundos, se refieren a la supervisión inefectiva y al dete- 
rioro de las condiciones económicas."

(Faraco, 1995: 11).

De lo planteado, se desprende que una de las causas fundamentales del deterioro de la gestión del sector bancario se debe a que la labor supervisora del ente encargado de controlar y regular el sistema bancario, denominado Superintendencia de Bancos ha sido inefectiva, al centrar su acción en aspectos formales del control, descuidando elementos referidos a la solvencia y calidad de la gestión; evidencia de ello, es que la gerencia de las entidades bancarias tomó decisiones no enmarcadas dentro de la normativa que regula las operaciones de la banca, mostrándose una posición de inobservancia de tal situación por parte del organismo fiscalizador.

La conclusión evidente la sintetiza (Faraco, 1995:14), cuando señala que: "Una supervisión inefectiva perpetúa políticas bancarias que hacen que la insolvencia adquiera dimensiones estructurales, o sea, que no pueden ser ya erradicadas por medios convencionales, sino mediante medidas radicales y dolorosas que, por su propia trascendencia, son cada vez más difíciles y costosas de adoptar".

La crisis financiera en el ámbito mundial ha dado lugar a que los paises se preocupen por introducir mecanismos de supervisión y control que permitan evaluar las operaciones que realiza la banca; tal evaluación deberá proporcionar información pertinente para tomar acciones que tiendan a superar las desviaciones que se presentan en el sector bancario, $y$ así alcanzar la normalidad que debe imperar en este tipo de instituciones. No obstante, se observa que aún existe en los gobiernos la incertidumbre de presentarse inconvenientes en el sistema en cuestión. Por tanto, resulta conveniente que el Ministerio de Hacienda promueva las acciones tendentes a evaluar el modelo que utiliza la Superintendencia de Bancos para diagnosticar las actividades del sector bancario venezolano, de forma tal que sea capaz de prever situaciones que pongan en peligro el sistema en cuestión. En particular, la banca comercial venezolana que asume hoy luego de la crisis bancaria reciente, la transformación a banca universal, la cual es más compleja por las múltiples operaciones que debe cumplir.

Diversos estudios se han realizado sobre los modelos de supervisión utilizados para evaluar la gestión de la banca comercial y universal.

En este sentido, Mujica y otros (1997) analizaron la situación financiera de la banca comercial en el periodo 1987-1994, en una muestra conformada por bancos intervenidos y no intervenidos de acuerdo a su influencia en el sistema bancario. El procedimiento utilizado consistió en realizar un análisis descriptivo para fijar las posiciones teóricas sobre el fenómeno social, determinando las variables e indicadores financieros relevantes para evaluar la situación financiera del sector, tomando como soporte los estados financieros; entre las conclusiones de la investigación destacaron que: la manera como se abordó el proceso de liberalización financiera constituyó el factor esencial que desencadenó la crisis financiera; la gerencia se caracterizó por actuar en el corto plazo; el proceso de supervisión y control fue inefectivo; la gerencia se dedicó a realizar operaciones 
especulativas de alto riesgo y los accionistas demostraron poca disposición para asumir riesgo de inversión.

En otra investigación, (Jiménez,1996) propuso una metodología para la evaluación financiera de los bancos comerciales en Venezuela, la cual se centró en desarrollar un modelo para la predicción de quiebra, trabajando con una muestra integrada por veinte (20) bancos comerciales con operaciones de carácter nacional, y llegó a las siguientes conclusiones: para analizar el comportamiento de los índices financieros tomados de los estados financieros de la banca comercial de Venezuela, el análisis discriminante es un instrumento útll y de ser utilizado el modelo propuesto por la Superintendencia de Bancos, el mismo puede contribuir al establecimiento de un sistema de alarma anticipada y a su vez permite exigir la adopción de correctivos necesarios, en el caso de que los resultados obtenidos indiquen una situación del banco con probabilidad de quiebra.

En esta misma línea de investigación, son coincidentes los hallazgos de (Valera, 1996) en el estudio de la aplicación del análisis discriminante para la clasificación de los bancos privados en Venezuela; la información para este estudio la constituyó el boletín trimestral de indicadores financieros de la banca comercial publicado por la Superintendencia de Bancos en el último trimestre de 1993. El análisis de los datos se llevó a cabo por computadora, utilizando el paquete estadístico para las ciencias sociales SPSS (Statistics Package for Social Sciencies). El estudio en cues- tión arribó a las siguientes conclusiones: la aplicación de la técnica del análisis discriminante llevó a la selección de aquellos indicadores que proveen un alto grado de separación en dos grupos sujetos a estudio, permitiendo además, la construcción de la función discriminante para hallar los puntajes que permitieron la clasificación de los casos para cada uno de los grupos, denominados bancos satisfactorios o bancos insatisfactorios.

\section{Principios básicos del modelo de supervisión bancaria}

Según información suministrada por el personal de la Gerencia de Supervisión de la Superintendencia de Bancos, las actividades de supervisión se orientan por los principios básicos siguientes:

- La supervisión estará enfocada hacia la evaluación de: a) los riesgos asumidos por las entidades sometidas al control de este Organismo, b) la calidad y eficacia de los sistemas implantados por las respectivas administraciones para gestionarlos y c) la capacidad patrimonial de las mismas para soportarlos; asimismo, debe ejercer una constante vigilancia y fiscalización del cumplimiento de la normativa vigente. La supervisión bancaria se caracterizará por ser un proceso proactivo y preventivo, con el propósito de permitir la identificación temprana de los problemas y la oportuna toma de decisiones bajo criterios uniformes, de acuerdo a la regulación prudencial, en pro del fortalecimiento del sistema bancario venezolano. 
- El carácter proactivo y prudencial de la supervisión bancaria se fundamentará en la Ley General de Ban$\cos$ y Otras Instituciones Financieras, asi como en el conjunto de regulaciones prudenciales que deben guiar y dar a conocer los criterios de supervisión adoptados por la Superintendencia, los cuales serán orientados por estándares internacionales y por la observancia de las mejores prácticas bancarias para identificar, medir y controlar los riesgos, a objeto de estimular y promover la autorregulación. Al mismo tiempo, esta normativa como parámetro referencial de los criterios de supervisión. debe permitir la adopción de las medidas correctivas a las desviaciones detectadas, de manera objetiva y sobre bases uniformes.

- La supervisión constituye un proceso continuo y coherente, que coordinará las diferentes actividades de la inspección "in situ" y la vigilancia "extra situ" con el uso o aplicación de métodos, técnicas y procedimientos de supervisión, de manera que permita un conocimiento integral de la situación económico-financiera de cada Institución, su tendencia y viabilidad; así como la transparencia de su gestión operativa y administrativa; se apoya en adecuados sistemas de información internos y en la estrecha comunicación que deberá mantenerse con las autoridades y auditores externos de cada Institución.

- La estrategia de supervisión individual para cada Institución deberá estar orientada por un sistema de calificación de bancos; clasificación ésta que será asignada al concluir cada visita general de inspección. Esta calificación reflejará en forma resumida el resultado del análisis y evaluación in situ, de aqueilos aspectos relacionados con la calidad de los activos y de los resultados obtenidos, 10 adecuado o no de los niveles de liquidez, los recursos propios para respaldar los riesgos asumidos y la calidad de los sistemas de control interno y de gestión para manejarlos, aplicándose este marco de análisis y evaluación de manera uniforme a todas las Instituciones. La calificación asignada definirá el plan de acción o conjunto de actividades supervisoras a ejecutarse en cada caso, permitiendo que la supervisión sea diferenciada entre una Institución y otra, de acuerdo con las circunstancias.

- El plan de acción o estrategia individual de supervisión deberá ejecutarse dentro de un ciclo de supervisión individual. El conjunto de estrategias individuales estructurará, a su vez, el ciclo de supervisión global a ser practicado al sistema bancario en general. Cada estrategia individual de supervisión considerará todas las actividades que se estimen adecuadas en función de la situación, tendencia, viabilidad, perfil de riesgo, calidad de la gestión y la importancia relativa que dentro del sistema bancario venezolano representa cada Institución y el grupo consolidable, según el caso. Cada estrategia individual de supervisión será diseñada con base en los resultados obtenidos en: a) la inspección in-situ y b) el análisis extra-situ, debiendo ser actualizada 
después de las visitas generales de inspección y monitoreada continuamente en el ínterin a fin de ajustarla a las nuevas circunstancias.

\section{Enfoque de supervisión}

El enfoque de supervisión adoptado en atención al proceso de reestructuración implementado en la Superintendencia de Bancos, ha sufrido transformaciones con el asesoramiento y ciertas pautas sugeridas por los organismos multilaterales (Fondo Monetario Internacional, Banco Mundial y Banco Interamericano de Desarrollo).

Con esta nueva visión, el proceso de supervisión se orienta al mejoramiento del proceso de planificación para un mayor conocimiento del negocio y áreas de riesgo y calidad de sus activos, a fin de lograr el desarrollo del proceso de inspección sobre bases de mayor objetividad en cuanto a la situación o condición de las instituciones financieras, identificando aquellas áreas o materias que requieren acciones correctivas por parte de la administración de las mismas, y desarrollar actividades de control y seguimiento, así como de la eficacia de las medidas correctivas tomadas para las referidas instituciones.

El proceso se realiza en forma integral, al considerar los aspectos claves que puedan afectar la estabilidad o solvencia de una institución financiera, tanto aquellos factores que puedan expresarse como indicadores cuantitativos, como los que requieren expresarse en juicios cualitativos, fundamentándose en las fortalezas o deficiencias especificas que se observan en una institución financiera.
Asimismo, el proceso de supervisión se ha dirigido a la ejecución de acciones preventivas, enfatizando el seguimiento a las áreas de debilidad identificadas o incentivando la adopción de medidas correctivas por parte de la administración del instituto.

A los fines de cumplir cabalmente con las funciones de supervisión y bajo el nuevo enfoque adoptado por la Gerencia de Supervisión de la Superintendencia de Bancos (Informe Anual, 1998), se ha orientado a desarrollar las siguientes acciones:

- Visita preliminar: El objetivo fundamental de esta visita es hacer una evaluación de la gestión realizada por los administradores de los institutos, a fin de determinar la calidad de las normas, políticas y controles internos establecidos en la organización para supervisar y controlar su adecuado funcionamiento.

Una vez concluido el desarrollo de la visita preliminar, se procede a elaborar la planificación de la visita final, apoyada con la resolución de los principales aspectos de evaluación, contemplados en un Manual de Inspección, implementado con informaciones y material de apoyo recibido de los Organismos Multilaterales y Organismos de Supervisión Bancaria de otros paises adaptados por esta Superintendencia, a las condiciones de nuestro mercado financiero.

- Visita final: Durante esta visita se efectúa una evaluación económicofinanciera de los Institutos sujetos a inspección. Para esto se toma como base los resultados obtenidos en la visita preliminar, así como también 
los obtenidos en la elaboración de la metodología para evaluar el riesgo bancario.

\section{Tipos de enfoques de supervisión}

El enfoque de supervisión se realiza bajo dos (2) modalidades fundamentales: inspección extra-situ e inspección in situ.

\subsection{Inspección extra situ (control indirecto)}

Esta actividad tiene como función principal analizar y evaluar toda aquella información suministrada por las instituciones financieras, según lo estipulado en el Ordenamiento Jurídico establecido por la Ley General de Bancos y Otras Instituciones Financieras, así como Normas Prudenciales, Resoluciones y Circulares emitidas por la Superintendencia de Bancos y Otras Instituciones Financieras.

Dentro de los objetivos de la Inspección Extra Situ (Control Indirecto), se tienen:

a. Mantener actualizados los expedientes correspondientes a los entes sometidos a control y supervisión de la Superintendencia de Bancos y Otras Instituciones Financieras.

b. Servir de apoyo al área de inspección para el proceso de elaboración de la planificación de las inspecciones a realizarse.

Para cubrir los objetivos planteados en esta inspección extra situ, la Superintendencia desarrolla las siguientes actividades:
- Evaluación económico-financiera de las Instituciones

La Superintendencia de Bancos ha dado prioridad a monitorear el comportamiento de las principales variables financieras del sector financiero, en especial, la banca comercial y universal. Al respecto ha delineado un plan de fortalecimiento dirigido a superar las debilidades del modelo de evaluación; para ello ha diseñado una metodología que permite clasificar el riesgo bancario, considerando en el diseño del modelo las categorías de análisis plasmadas en el modelo CAMEL, desarrollado por el Comité de Basilea de regulaciones Bancarias y Prácticas de Supervisión (1988):

- C: Capital Adecuacy (Suficiencia de Capital)

- A: Assets Quality (Calidad de Activos)

- M: Management

(Gestión Administrativa)

- E: Eaming (Rentabilidad)

- L: Liquity (Liquidez)

La metodología para evaluar el riesgo, diseñada por el Departamento de Estadística adscrito a la Gerencia Técnica de la Superintendencia de Bancos (1998), se concentra en la selección previa de los indicadores más relevantes para medir el comportamiento histórico de la situación financiera de la banca comercial y universal; este análisis proporciona información pertinente sobre la solidez, eficiencia y rendimiento de cualquier institución financiera. La clasíficación del riesgo bancario en términos de su desempeño, se desagrega en dos grupos: Satisfactorio e Insatisfactorio y dentro de cada grupo, los bancos se definen como AAA, AA-Plus, AA, A y $O$. 
El procedimiento que se utiliza para determinar los dos grupos consiste en lo siguiente:

El desempeño de cada banco se mide con base en la valoración que va de uno a cinco puntos, o su equivalente alfabético que va desde AAA, AA-Plus, AA, A y 0 . Expresándose: $5=\mathrm{AAA}, 4=\mathrm{AA}$ Plus, $3=A A, 2=A$ y $1=0$

Donde:

5 = AAA: Lo constituyen los bancos sanos, con una fortaleza en casi todos sus aspectos, por no tener áreas del negocio de preocupación; sin embargo pudieran presentar problemas insignificantes. 4 = AA-Plus: Constituido por los bancos saludables, aunque pudieran reflejar debilidades moderadas, susceptibles de subsanar en el curso normal de su actividad.

$3=\mathrm{AA}$ : Lo constituyen los bancos que tienen problemas financieros, operacionales o de cumplimiento de normas, en una escala que varía de moderado a insatisfactorio; presentan vulnerabilidad a los cambios del entorno. Si la gerencia no toma acciones correctivas podrian tener un deterioro acelerado. En esta categoría se precisa un seguimiento e inspección para corregir las deficiencias señaladas. 2 = A: Constituido por los bancos que muestran excesivas debilidades en el activo o una combinación de limitaciones que generan resultados menos satisfactorios. Los problemas que presentan podrian comprometer su supervivencia.

$1=0$ : Constituyen los bancos que poseen debilidades fuertes, que podrían ir a una quiebra, por lo que se requiere ayuda de parte de los accionistas o de otras fuentes para superar sus problemas financieros, ya que existen suficientes elementos para inferir una alta probabilidad de fracaso o quiebra.

De la combinación de las escalas o valores señalados, se determina lo siguiente:

$0=A A+A+O$ Bancos con desempeño insatisfactorio

1 = AAA + AA-Plus Bancos con desempeño satisfactorio

En este orden de ideas, la Superintendencia asigna una valoración a los distintos niveles de desempeño, tal como se expresa en la Tabla $N^{\circ} 1$.

Para la determinación de la calificación global de riesgo en cada uno de los Bancos Comerciales y Universales, se asignan valores promedio por la cualidad del desempeño obtenido. Tal como se indica en las Tablas $\mathrm{N}^{\circ} 2$ y 3 .

La evaluación y clasificación de riesgo se realiza a través de diez (10) indicadores seleccionados como relevantes por la Superintendencia, los cuales le permitirán inferir sobre el desempeño de los bancos comerciales y universales. Determinado el valor de los indicadores financieros de cada banco, se le ubica según las respectivas calificaciones en la categoria respectiva ( $M B, B, A, M)$, de acuerdo a los intervalos diseñados. Tal como se señala en la Tabla $N^{\circ} 3$ :

La metodología descrita, establece valores por cada indicador financiero, 10 que permite conocer un puntaje en función de las categorías antes señaladas, lo cual conlleva a precisar cuáles bancos son sólidos y eficientes o en su defecto presentan problemas en su desempeño. 
Tabla 1

Banca Comercial y Universal en Venezuela

Valoración de riesgo por niveles de desempeño

\begin{tabular}{cll} 
Código & Categoria & Calificación de riesgo \\
\hline 5 & AAA & Mayor o igual a 70 puntos \\
4 & AA-Plus & $60<=$ AA-Plus $<70$ \\
3 & AA & $50<=$ AA $<60$ \\
2 & A & $30<=\mathrm{A}<50$ \\
1 & 0 & $0<30$ \\
\hline
\end{tabular}

Fuente: Departamento de Estadística de la Gerencia Técnica de la Superintendencia de Bancos.

\section{Tabla 2}

Banca Comercial y Universal en Venezuela Clasificación global del riesgo por cualidad del desempeño

\begin{tabular}{lc} 
Categorias (cualidad) & Puntaje \\
\hline Muy Bueno (MB) & 10 \\
Bueno (B) & 6 \\
Regular (R) & 3 \\
Malo (M) & 0 \\
\hline
\end{tabular}

Fuente: Departamento de Estadistica de la Gerencia Técnica de la Superintendencia de Bancos.

- Análisis de los informes de auditores externos

Los dictámenes sobre los estados financieros y carta de gerencia emitidos por los Auditores Externos de las entidades financieras se utilizan como complemento en el análisis de la situación económico financiera de los institutos, para corroborar situaciones detectadas, y en caso de incongruencia, aplicar bases legales para las discusiones técnicas.

\section{- Control patrimonial}

Esta actividad permite realizar un seguimiento a la evolución de la incidencia de los resultados de las últimas ins- pecciones realizadas a los institutos, haciendo mayor énfasis para los análisis de reparto de dividendos y/o aumentos de capital, según los planes de ajustes de adecuación de capital mínimo establecido en la Ley General de Bancos y Otras Instituciones Financieras.

- Revisión de las actas de las asambleas ordinarias y extraordinarias de accionistas

En esta actividad se revisan los recaudos que son presentados por las Juntas Directivas a los accionistas de las instituciones financieras, en ocasión de la celebración de las referidas asambleas. 


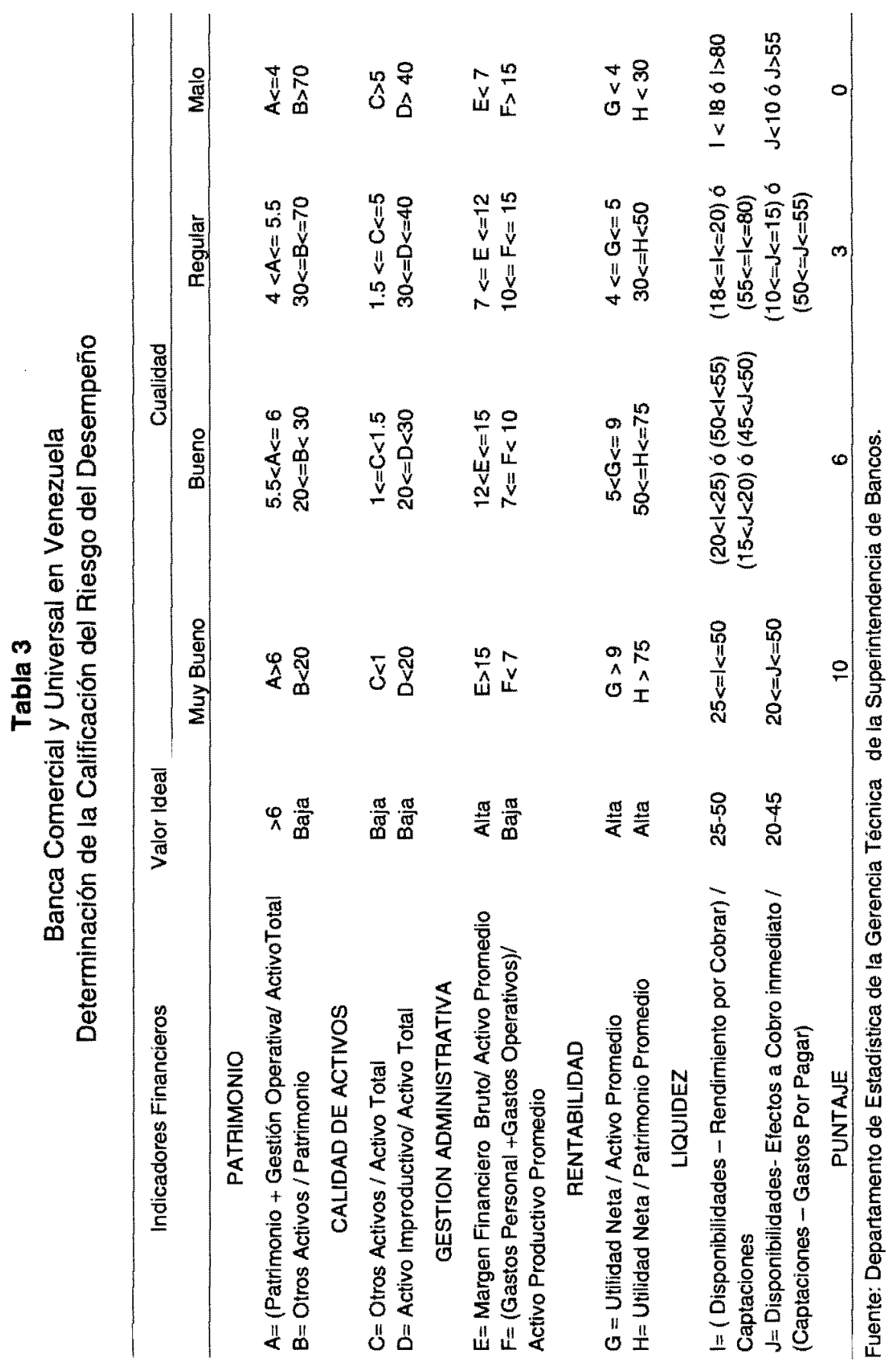


señalándose las irregularidades detectadas e igualmente formulándose las recomendaciones pertinentes en cada caso.

\subsection{Inspección in situ (control directo)}

Este control se ejerce en las sedes principales de los institutos supervisados, dando cumplimiento a lo establecido en la Ley General de Bancos y Otras Instituciones Financieras. Las instituciones financieras a ser inspeccionadas se seleccionan fundamentándose en:

- Los resultados obtenidos de la evaluación y análisis de la situación económico-financiera, que determinan factores de riesgos de la institución, incidiendo en su solvencia y liquidez.

- Complejidad y magnitud de los entes supervisados.

- Antigüedad de las inspecciones anteriores, de acuerdo al ordenamiento legal de realizar por lo menos una inspección a cada instituto una vez al año.

Para cubrir los objetivos planteados en esta inspección in situ, la Superintendencia desarrolla las siguientes actividades:

\section{a. Inspecciones generales}

Las visitas de inspección general se realizan una vez al año; constituyen una de las funciones más importantes de supervisión, toda vez que se realiza una evaluación de los activos de riesgo, fideicomiso, mesa de dinero, control interno, y por ende una visión de conjunto sobre la administración de la institución financiera.

\section{b. Visitas especiales}

Las visitas especiales se efectúan con el fin de verificar y examinar aspec- tos específicos de las operaciones realizadas por los institutos, el seguimiento de la normalización de observaciones detectadas, denuncias formuladas por el pübli$\mathrm{co}$, etc.

\section{Evaluación de Riesgo en la Banca Comercial y Universal}

Para analizar su validez, se aplicó la metodologia de supervisión extra situ utilizada por la Superintendencia de Bancos para evaluar el riesgo del sector bancario: para ello se consideró como población la Banca Comercial y Universal de Venezuela, en el periodo comprendido desde el primer semestre de 1996 hasta el primer semestre de 1998. La población fue sometida a un proceso de evaluación previa para evitar sesgos en los resultados que pudieran inferirse en el estudio, se tomaron como criterios que los sujetos sociales deben realizar operaciones en el ámbito nacional, con información completa en todos los periodos señalados $y$ que reúnan características especiales en cuanto a su situación financiera.

Se consideró como criterio para la selección de la muestra, las entidades bancarias con mayor volumen de captación de ahorros, pudiéndose determinar siete(7) bancos cuyas captaciones representan más del setenta y cinco $(75 \%)$ del total de captación nacional dentro del sistema bancario. Este constituye el indicador utilizado por el Banco Central de Venezuela para seleccionar aquellos bancos de mayor relevancia en el sector bancario y poder asi establecer las tasas activas y pasivas que regirán en el mercado. En este sentido, la muestra quedó conformada por los siguientes bancos comer- 
ciales y universales: Provincial, Venezuela, Mercantil, Corp Banca, Unión, Industrial de Venezuela y Caribe, cuyos estados financieros obtenidos a través de la Superintendencia de Bancos fueron sometidos al análisis de riesgo.

En la Tabla 4 se observa el comportamiento que asume la valoración del riesgo de la banca comercial y universal durante el período objeto de estudio, resultado obtenido de la aplicación de la metodología para evaluar la situación económico-financiera, considerando como variable determinante el riesgo. A continuación se describe la tendencia del riesgo para cada una las instituciones seleccionadas en la muestra.

- El Banco Provincial mostró un comportamiento uniforme en la valoración del riesgo, situándose en AA-Plus en la mayoría de los semestres estudiados, excepto en el primer semestre de 1996 que se ubicó en $\mathbf{A}$.

- El Banco de Venezuela reflejó un comportamiento moderado y uniforme en la valoración del riesgo, situándose en AA en la mayoria de los semestres estudiados, excepto en el segundo semestre de 1996 que se ubicó en AA-Plus.

- El Banco Mercantil mostró un comportamiento uniforme en la valoración del riesgo, situándose en $\mathrm{A}$ en la mayorla de los semestres estudiados, excepto en el segundo semestre de 1996 que se ubicó en AA.

- Corp Banca mostró un comportamiento moderado y uniforme en la valoración del riesgo, situándose en AA en los tres primeros semestres y en
AA-Plus en los dos últimos semestres analizados.

- El Banco Unión mostró un comportamiento alternado en la valoración del riesgo, situándose en A y AA.

- El Banco Industrial de Venezuela mostró un comportamiento uniforme en la valoración del riesgo, situándose en $A$, excepto en el primer semestre de 1996 que se ubicó en AA.

- El Banco Caribe mostró un comportamiento excepcional en la valoración del riesgo, situándose en AA en el primer semestre de 1996, en AAA en el segundo semestre de 1996, en AAPlus en los semestres primero y segundo de 1997 y AA en el primer semestre de 1998.

\section{Conclusiones}

De los resultados obtenidos se destacan las siguientes conclusiones:

- La banca comercial y universal venezolana muestra una valoración en el riesgo calificada como moderada y medianamente alta, lo cual imposibilitará en el corto plazo la superación de la crisis que aún persiste en el sector.

- La metodología de evaluación aplicada para determinar el riesgo deberá ser complementada con modelos estadisticos y matemáticos, tales como el modelo de regresión logística y el análisis discriminante, los cuales permiten predecir con una mejor efectividad las debilidades financieras que pudieran presentarse en las instituciones del sector bancario. 


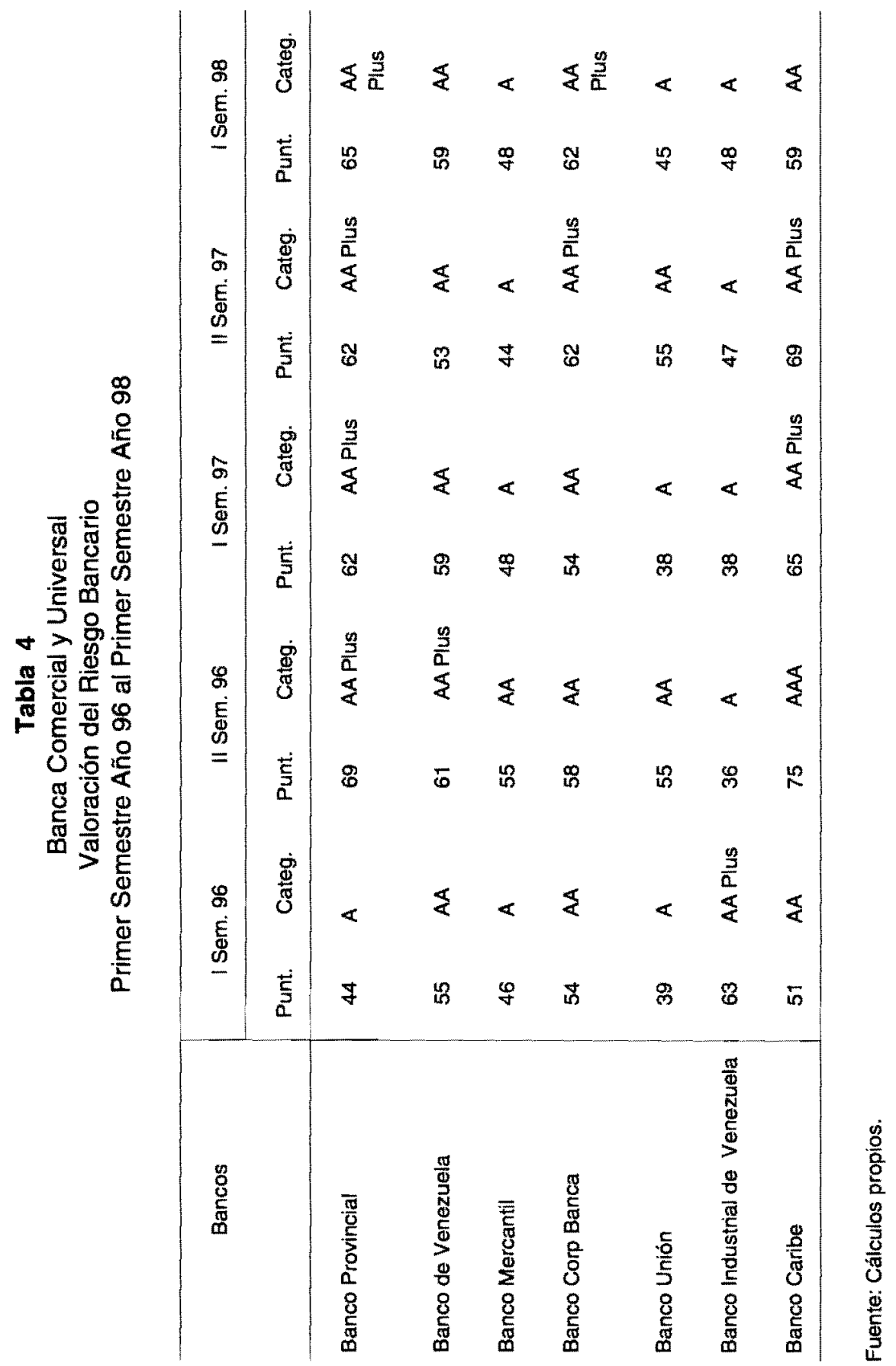




\section{Bibliografía citada}

Faraco, Francisco y Suprani Romano (1995). La Crisis Bancaria Venezolana. Análisis Preliminar. Editorial Panapo. Caracas. Venezuela.

Jiménez M., Edinson E. (1996). Metodología para la Evaluación Financiera de los Bancos Comerciales en Venezuela. Modelo para la Predicción de Quiebra. Trabajo de Ascenso presentado para optar a la categoria de Profesor Titular.

Mujica F., Aníbal (1995). "Liberación Financiera y Crisis Bancaria", en Revista de Ciencias Sociales. FCES - LUZ, Nueva Epoca. Vol. 1, No. 2. Maracaibo. Venezuela.
Valera M., José R. (1996). Aplicación del Análisis Discriminante para la Clasificación de los Bancos Privados en Venezuela. Trabajo de Ascenso para optar a la categoria de Profesor Asistente. Universidad Central de Venezuela. Caracas. Venezuela.

Superintendencia de Bancos (1998). Metodología para la Clasificación de Riesgo de los Bancos Comerciales y Universales según su Desempeño. Caracas. Venezuela.

Superintendencia de Bancos. Informe Anual 1997. Caracas. Venezuela.

Superintendencia de Bancos. Entrevistas al Personal de la Gerencia de Supervisión. 